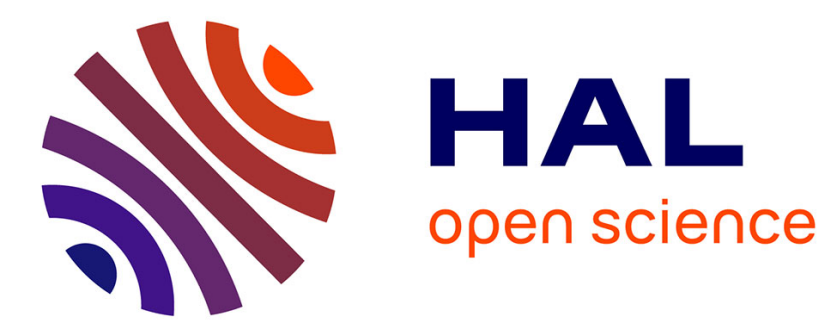

\title{
XY model with weak random anisotropy in a symmetry-breaking magnetic field
}

Bernard Dieny, Bernard Barbara

\section{To cite this version:}

Bernard Dieny, Bernard Barbara. XY model with weak random anisotropy in a symmetry-breaking magnetic field. Physical Review B: Condensed Matter and Materials Physics (1998-2015), 1990, 41 (16), pp.11549 - 11556. 10.1103/PhysRevB.41.11549 . hal-01660291

\section{HAL Id: hal-01660291 https://hal.science/hal-01660291}

Submitted on 10 Dec 2017

HAL is a multi-disciplinary open access archive for the deposit and dissemination of scientific research documents, whether they are published or not. The documents may come from teaching and research institutions in France or abroad, or from public or private research centers.
L'archive ouverte pluridisciplinaire HAL, est destinée au dépôt et à la diffusion de documents scientifiques de niveau recherche, publiés ou non, émanant des établissements d'enseignement et de recherche français ou étrangers, des laboratoires publics ou privés. 


\title{
$X Y$ model with weak random anisotropy in a symmetry-breaking magnetic field
}

\author{
Bernard Dieny and Bernard Barbara \\ Laboratoire Louis Neel, Centre National de la Recherche Scientifique, 25 Avenue des Martyrs, \\ Boite Postale No. 166X, 38042 Grenoble CEDEX, France
}

(Received 18 September 1989)

\begin{abstract}
We present a numerical study of the two-dimensional classical $X Y$ model with weak random anisotropy at zero temperature. Zero-field configurations obtained by ultrafast cooling, firstmagnetization curves, and hysteresis loops have been calculated for different random-anisotropyto-exchange ratios. In zero field, a pinning of vortices by the random-anisotropy field occurs. It prevents the binding then collapsing of pairs of opposite charges and thus leads to a nonferromagnetic ground state. Applying a magnetic field causes a progressive depinning of vortices that disappear in pairs until saturation. Starting from saturation and decreasing the applied field leads, in zero-field, to a magnetic state of large remanent magnetization. However, an important aftereffect is observed. It should give smaller remanence after much longer computer time. Then, the reversal of magnetization in negative fields occurs through a peculiar process that involves the formation and collapse of new kinds of topological defects (infinite strings). These linear defects are in fact the ultimate stage in the shrinking of domains oriented in the initial direction of saturation. Their collapse occurs abruptly through the creation and propagation in opposite directions, along the defect, of an unbound vortex-antivortex pair.
\end{abstract}

\section{INTRODUCTION}

Both the classical isotropic $X Y$ model and the magnetic properties of random-anisotropy systems have attracted great interest during the last decade. Very recently it was established under very general conditions that conventional long-range order is destroyed in two-dimensional (2D) systems with continuous symmetry at finite temperature. This applies, for example, to $2 \mathrm{D}$ crystals, ${ }^{1} \mathrm{mag}$ nets, ${ }^{2}$ superfluids, and superconductors. ${ }^{3}$ Later Kosterlitz and Thouless (KT) dealt with these systems within a unified theoretical model. ${ }^{4}$ They contend that the 2D low-temperature phase is characterized by a power-law decay in the pair-correlation function with a temperature-dependent exponent. This phase is characterized by the presence of pairs of tightly bound defects of opposite signs, which are dislocations in crystals, spin vortices in magnets, and quantum vortices in superfluids. At a characteristic temperature $T_{K L}$, the system undergoes a phase transition in which the pairs of defects unbind to create a new phase where the correlations decrease exponentially. The KT prediction for the 2D superfluid, superconductor, and $X Y$ models has been confirmed by various experiments and computer simulations. ${ }^{5}$ Moreover, several extensions of the pure classical $X Y$ model have been studied. ${ }^{6-11}$ In particular, effects of weak random Dzyaloshinskii-Moriya interactions ${ }^{8}$ or of random $p$-fold symmetry-breaking fields, ${ }^{9-11}$ have been investigated. In these models a vortex unbinding mechanism has been found within the low-temperature phase as a result of a pinning or a screening of their interactions by the random field.

Magnetic properties of random-field systems (randomanisotropy fields of continuous symmetry, $p$-fold symmetry-breaking fields, random-exchange fields, etc.) have been studied theoretically and experimentally in 3D and sometimes $2 \mathrm{D}$ systems. Imry and $\mathrm{Ma}^{12}$ and later on Pelcovits, Pytte, and Rudnick ${ }^{13}$ showed that an arbitrary weak random field destroys ferromagnetic order in a system with continuous symmetry (like Heisenberg or $X Y$ magnets) below four spatial dimensions. Then, by using a Green-function formalism, Chudnovsky, ${ }^{14,15}$ Saslow, and Serota ${ }^{16}$ have investigated magnetic properties of $2 \mathrm{D}$ amorphous magnets with local random anisotropy and applied magnetic field. Expressions for the angle-angle correlation function and for the magnetization law have been derived. The zero-field-cooled phase (called correlated spin glass) is characterized by smooth and stochastic rotations of the magnetization over the sample. However, topological defects specific of $2 \mathrm{D}$ systems have not been explicitly introduced in their calculation.

In this paper we present a numerical study at zero temperature of the classical 2D $X Y$ model with weak random anisotropy and in the presence of an applied magnetic field (dipolar effects are not taken into account). After an ultrafast cooling in zero field the low-temperature phase exhibits both Imry and Ma domains and vortices which are pinned by the random anisotropy field and thus do not disappear at $T=0$. Then, applying a magnetic field leads to a progressive depinning of vortices which bind by pair and progressively disappear until saturation. At this pairing and collapsing field-induced mechanism is superposed a progressive polarization of magnetic moments towards the field direction (ferromagnet with wandering axis $\left.{ }^{16}\right)$. Decreasing the applied field from saturation leads to a phase of large remanent magnetization in which the magnetization varies continuously in a semicircle oriented along the saturation field. This phase does not contain any vortex. However, the dynamics of relaxation in this decreasing field procedure is rather slow and a large 
aftereffect is observed in zero field. As a result, a much longer computer run should lead to a state of lower remanent magnetization. When a negative field is applied, a new kind of topological defect is observed. These defects look like infinite strings and are characterized by a linear core in which the magnetization is trapped in the initial field direction (saturation field direction) and by a magnetization parallel to the applied field on their border. The width of the string decreases down to a few interatomic distances when the strength of the field increases sufficiently. Above a certain critical field the string abruptly collapses through the creation and propagation along the defect of a vortex-antivortex pair.

This paper is divided into four sections. The first one gives some indications on the numerical method. The second deals with a comparison of zero-field configurations obtained by ultrafast cooling from the paramagnetic state for different values of the randomanisotropy constant $D$. In the third one, we present the first magnetization curves obtained by starting from the zero-field states discussed in the second part. The last section concerns hysteresis loops with a particular emphasis on the infinite-string defects observed in negative fields.

\section{NUMERICAL METHOD}

All the calculations are carried out at zero temperature on a square lattice of $100 \times 100$ spins with cyclic boundary conditions. The Hamiltonian is

$$
\mathscr{H}=-J \sum_{\langle i, j\rangle} \mathbf{S}_{i} \cdot \mathbf{S}_{j}-D \sum_{i}\left(\mathbf{n}_{i} \cdot \mathbf{S}_{i}\right)^{2}-\mathbf{H} \cdot \sum_{i} \mathbf{S}_{i},
$$

where $\langle i, j\rangle$ indicates a summation on nearest neighbors, $\mathbf{n}_{i}$ is a unit vector of the local atomic anisotropy axis, $J$ is the exchange coupling constant, $D$ is the uniaxial anisotropy constant, and $\mathbf{H}$ is the applied magnetic field. A cycle in the calculation consists of exploring all of the 10000 spins in a random order and setting each explored spin in its local minimum of energy between its anisotropy axis and local-field direction. For each value of the parameters $D$ and $\mathbf{H}$ the calculation was run until the greatest variation of angle of a spin between two consecutive cycles was smaller that $5 \times 10^{-4} \mathrm{rad}$. As pointed out by Tobochnik and Chester, ${ }^{17}$ starting from a random configuration and running the calculation at very low temperatures generates metastable states of higher energy than those obtained using a slow-cooling procedure. Thus, for instance, the configurations we obtain in zero field after ultrafast cooling exhibit more vortices than they should if a slow zero-field cooling had been realized. This may be somewhat disturbing in a quantitative point of view but is not a real problem in this paper, the main purpose of which is to show some qualitative features of the magnetization processes in these $2 \mathrm{D}$ systems with random anisotropy.

\section{ZERO-FIELD CONFIGURATIONS}

Figure 1 shows two configurations obtained for the same set of random-anisotropy axes but two different values of $D / J(D / J=0.1$ and 0.6$)$. They have a small
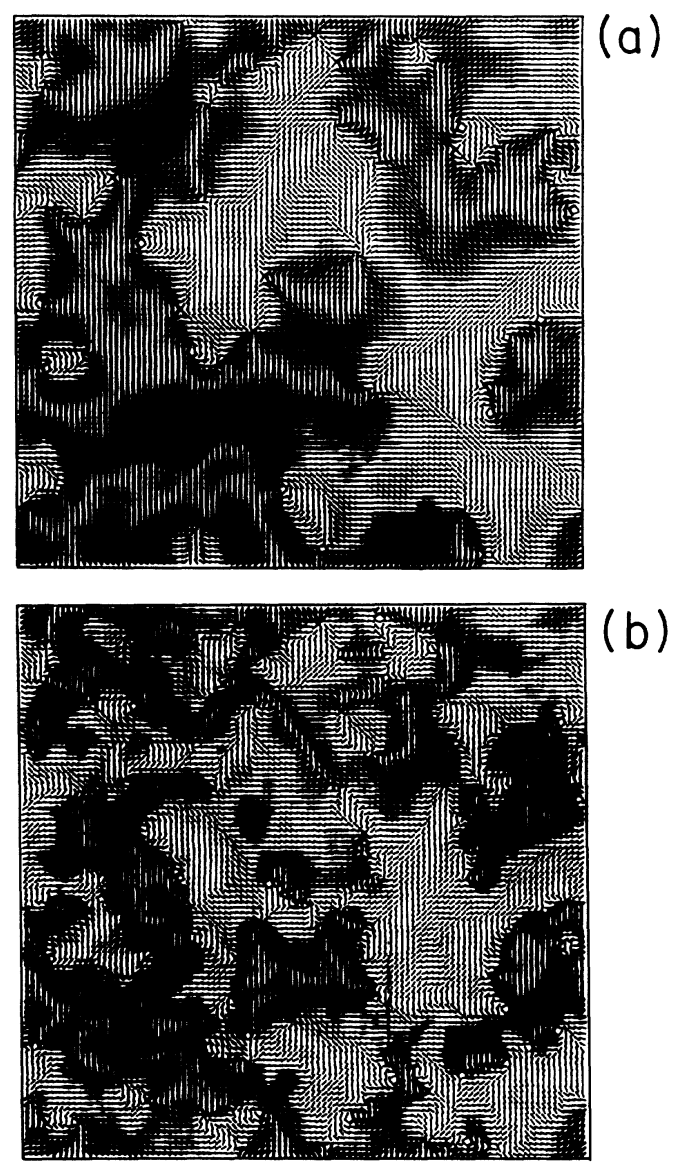

FIG. 1. Two spin configurations obtained for the same set of random-anisotropy axes but two different values of the random-anisotropy-to-exchange ratio $D / J$. (a) $D / J=0.1$. (b) $D / J=0.6$. The different thicknesses of the arrows simply indicate the spins pointing in the upper (respectively lower) hemicircle.

net magnetization and contain regions with local ferromagnetic order (Imry and Ma domains ${ }^{12}$ ) the size of which decreases with $D / J$. These domains are not specific of two dimensions; they have already been studied theoretically and experimentally in 3D randomanisotropy systems. ${ }^{16,18,19}$ The small resultant magnetization observed here decreases with $D / J$ and can be ascribed to the finite size of the sample and thus finite number of Imry and Ma domains. Indeed, if the sample is divided into $n$ ferromagnetic Imry and Ma domains randomly oriented in the $X Y$ plane, then a random-walk argument gives a resultant magnetization $M$ of the order of $(n)^{-1 / 2}$ which is in agreement with our results. Figure 1 also shows the existence of stable topological defects, namely vortices and antivortices, despite that the system is at zero $\mathbf{K}$. The Kosterlitz-Thouless theory of the pure $X Y$ model, ${ }^{4}$ as well as numerical simulations, ${ }^{17,20}$ show that a pairing mechanism between vortices of opposite charges occurs at and below the KT transition. At the thermal equilibrium the number of vortices is determined by a counterbalance between vortex-antivortex annihilations and vortex-antivortex pair creations by thermal ac- 
tivation. At absolute zero temperature, these topological defects disappear in the pure $X Y$ model, leading to a completely ferromagnetic ground state. This picture has to be modified in the presence of random anisotropy, where we observe that stable vortices persist at $T=0 \mathrm{~K}$. As a matter of fact, the random-anisotropy field, although preserving the continuous symmetry (at least at scale larger than Imry and Ma domains), creates local energy barriers preventing easy spin rotations and therefore preventing the motion of vortices of opposite sign towards each other. Furthermore, it introduces a characteristic cutoff length in the system which is of the order of the 2D Imry and Ma domain size $\sim(J / D)$. As a result, the number of vortices that the sample can contain should increase with $D / J$. This is effectively observed: the number of vortices of each sign is equal (periodic boundary conditions) and increases rapidly with $D / J$, especially around $D / J \sim 0.4$. For $D / J<0.2$ and $D / J>0.8$, it tends to become constant (Fig. 2). A saturation of the number of vortices for large $D / J$ can be expected in such a system because (i) in the limit of infinite anisotropy the size of Imry and Ma domains tend to be independent of $D / J$ (a few interatomic distances) and (ii) the pinning of vortices will be maximum for $D \gg J$, and therefore not very sensitive on a further increase of $D / J$. The finite and nearly constant number of vortices obtained for low $D / J$ cannot be explained on the basis of "normal" metastable equilibrium due to the topological disorder of random anisotropy. In fact, the dynamics for the annihilation of vortex-antivortex pairs becomes extremely slow when the number of vortices tends to zero. The vortice number decays at a rate proportional to the number of pairs, so that exponential annihilation results. ${ }^{20}$ Consequently a very large computer time would be necessary to complete total annihilation of vortice pairs in the limit $D=0$.

At finite $D$, i.e., in the presence of disorder, it is possible to identify some local configurations of anisotropy axes as pinning centers for vortices. In particular, a small area over which the set of anisotropy axes has the same cylindrical symmetry as a vortex constitutes an energetically favorable location for a vortex and then is a

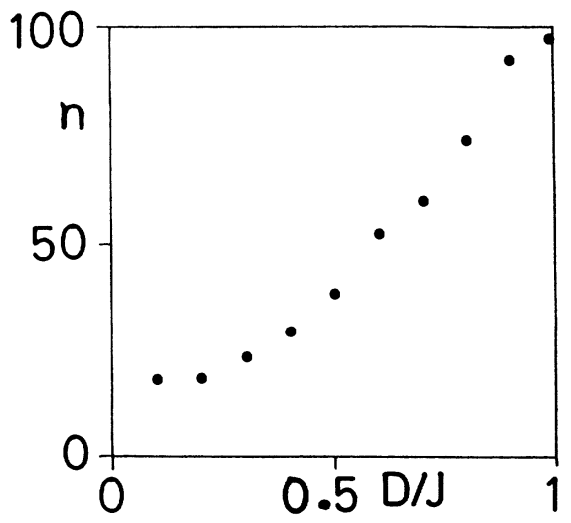

FIG. 2. Number of vortices $n$ of each sign in the zero-field configuration vs $D / J$ for the same set of anisotropy axis. pinning center. As an illustration of that, it can be seen in Fig. 1 that some vortices are located exactly at the same place for different values of $D / J$ but the same set of anisotropy axes. The trapping of vortice configurations by disorder has already been discussed in several theoretical studies. ${ }^{8-11}$ In particular, Rubinstein et al. ${ }^{8}$ have shown that a two-dimensional $X Y$ magnet with random Dzyaloshinsky-Moriya interactions is equivalent to the usual neutral Coulomb gas of vortex charges ${ }^{4}$ but coupled to a quenched distribution of dipoles. Below a characteristic freezing temperature, the vortex pairs are ripped apart by the random potential generated by the quenched dipole array. Similarly, Goldschmidt and Schaub, ${ }^{11}$ considering the $X Y$ model with random $p$-fold anisotropy, showed that an unbinding of vortices also occurs at low temperature which renders the glass phase paramagnetic at large scales. As described above, such an unbinding mechanism due to disorder is clearly seen in our simulations.

In other respects, it is worthwhile to notice that the spatial repartition of vortices is not homogenous over the sample (see Fig. 3). The vortices tend to bind by pairs but also to agglomerate. This joins the point underlined by Tobochnick and Chester ${ }^{17}$ at finite temperature, according to which, the more vortices existing on an area of the sample, the easier it is to add other vortices in this same area. In other words, considering vortices as particles, the local chemical potential decreases with the density of vortices.

Let us now go further by considering the two-spin pair correlations (see Fig. 4). The spin configurations show that $\left\langle\mathbf{S}_{i} \cdot \mathbf{S}_{j}\right\rangle$ decreases rapidly with distance, this decrease being more pronounced for large $D / J$. This is a general feature in 3D random-anisotropy systems because the size of Imry and $\mathrm{Ma}$ domains ${ }^{1}$ decreases with $D / J$ $\left[1 \sim(J / D)^{2}\right.$ in three dimensions]. In 2D systems such domains can also be defined (although $d=2$ is a marginal

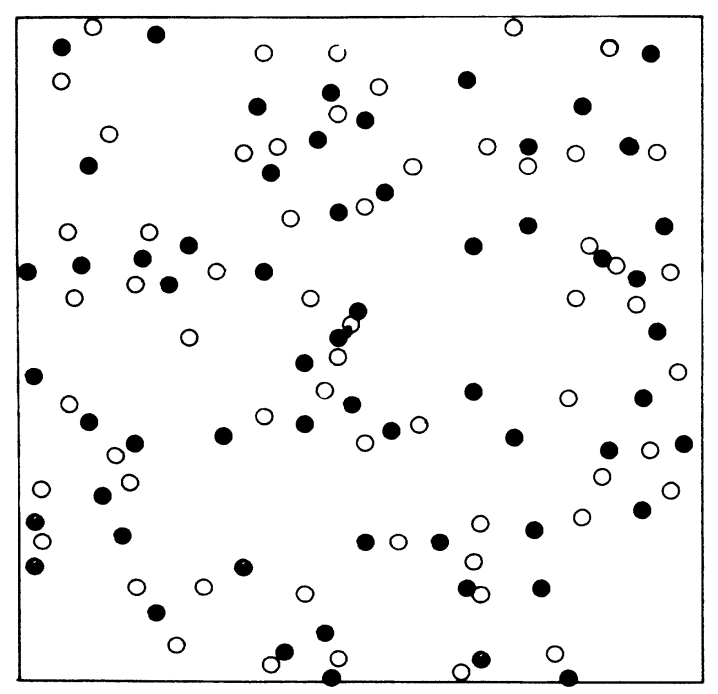

FIG. 3. An example of spatial repartition of vortices for $D / J=0.6$ [same spin configuration as in Fig. 1(b)]. The color indicates the charge of the vortices. 

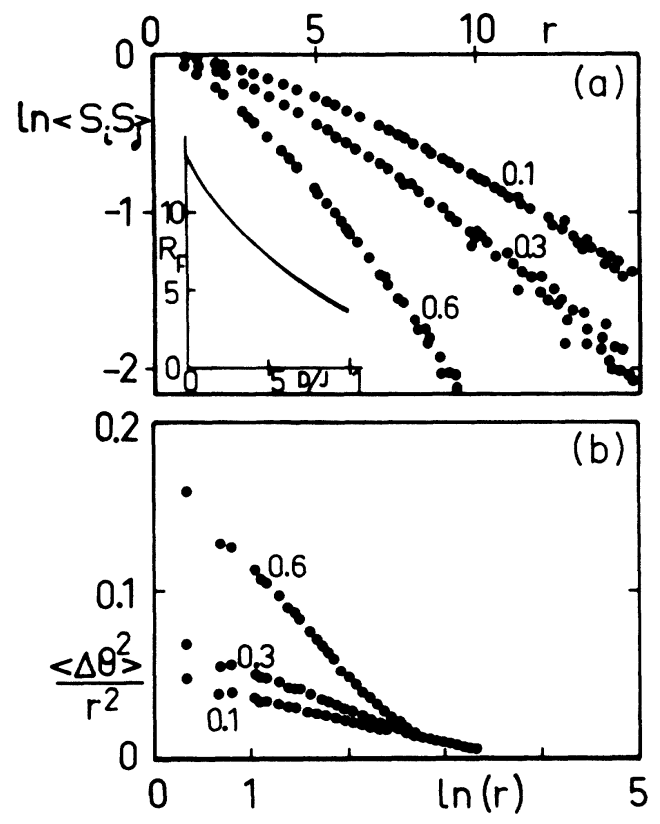

FIG. 4. Spin-spin correlation function $\ln \left\langle S_{i} S_{j}\right\rangle$ vs $r$ for three values of $D / J: 0.1,0.3$, and 0.6. Inset: Correlation length $R_{F}$ vs $D / J$. (b) Angle-angle correlation function $\left\langle\left(\theta_{i}-\theta_{J}\right)^{2} / r^{2}\right\rangle$ vs $\ln r$ for comparison with the formula given Ref. 14 (formula $I$ in the text).

dimensionality for straightforward implementation of the qualitative Imry and $\mathbf{M a}^{12}$ argument) and their size also decreases with $D / J[1 \sim(J / D)$ in two dimensions]. However, the observed rate of the decrease of $\left\langle\mathbf{S}_{i} \cdot \mathbf{S}_{j}\right\rangle$ is rather influenced by the presence of vortices and also by the fact that our spin configurations have a high degree of metastability. In order to get some insights into the role of vortices and metastability, we have compared angleangle correlations calculated directly from angle differences with the expression found, in the absence of vortices, by Chudnovsky: ${ }^{14,16}$

$$
\left\langle\left(\theta_{i}-\theta_{j}\right)^{2}\right\rangle=\frac{1}{4}(D / J)^{2}(r / a)^{2} \ln |L / r|,
$$

where $r=\left|\mathbf{r}_{i}-\mathbf{r}_{j}\right|$ and $L$ is the size of the system. Interestingly, the plot of $\left\langle(\Delta \theta)^{2}\right\rangle / r^{2}$ versus $\ln r$ shows an almost linear behavior for values of $r$ not too close to the interatomic distance, and this despite the presence of vortices and metastability. In fact, this agreement with (1) is only qualitative: the intercept of the line of negative slope with the abscissa is not a constant which should be equal to the size of the sample but takes a much smaller value and is a function of $D / J$, which can be put on the phenomenological form $L_{c}=40 e^{-1.4 D / J}$. Furthermore, the slope, although increasing with $D / J$, is smaller than $\frac{1}{4}(D / J)^{2}$, if $D / J$ is not too small. For small $D / J$, we observe a constant and finite slope due to the vortices persisting when $D \rightarrow 0$ (already discussed). Thus, the presence of vortices and metastability seems to reduce drastically ferromagnetic correlations for weak random anisotropy. For large values of $r$, another regime independent of $D / J$ is observed [Fig. 4(b)]. This simply indicates that the assumption of small-angle rotation in formula (1) is no longer valid. Indeed, for large $r$, if all angles are taken between 0 and $2 \pi$, then $\left\langle\left(\theta_{i}^{2}-\theta_{j}^{2}\right)\right\rangle \sim \pi^{2}$ because of the absence of long-range order. The plot of Fig. $4(\mathrm{~b})$ is then equivalent to $\pi^{2} / r^{2}$ versus $\ln r$ which is independent of $D / J$.

Now, from the plot of Fig. 4(a), it is possible to derive a ferromagnetic correlation length $R_{F}$ by the distance over which $\left\langle\mathbf{S}_{i} \cdot \mathbf{S}_{j}\right\rangle$ decreases from 1 to $\exp (-1)$. This correlation length is plotted versus $D / J$ in the inset of Fig. 4(a). We note that, within error bars, $R_{F}$ is proportional to the cutoff length $L_{c}$ found in Fig. 4(b):

$$
R_{F}(D / J) \approx 0.35 L_{c}(D / J) \approx 14 e^{-1.4 D / J} .
$$

As already discussed, $R_{F}$ does not diverge in the limit $D / J \rightarrow 0$ because of the slowing down of the dynamics for the low $D / J$ ratio preventing the complete disappearance of vortices in our calculation. It would be worthwhile to calculate the correlation length for $D / J \rightarrow 0$ starting from a completely disordered state at different states of the relaxation process, i.e., as the number of vortices decreases down to zero. This would be useful in understanding the quantitative influence of vortices on the ferromagnetic correlation length.

\section{FIRST MAGNETIZATION CURVE}

Starting from one of the zero-field configurations described above, a magnetic field is applied in the $X Y$ plane. The main characteristics of the first magnetization curves (see Fig. 5) are the following: in average, positive curvature in low fields and negative curvature in large fields, existence of more or less important magnetization jumps. From a qualitative point of view, two kinds of magnetic processes contribute to the observed magnetization curve.

(1) The first one is a progressive depinning of vortices by the applied field. The field helps the magnetization to overcome the energy barriers due to random anistropy and then causes the motion of vortices of opposite charges towards one another. When two vortices become sufficiently close they annihilate. This more or less abrupt annihilating gives a jump on the magnetization

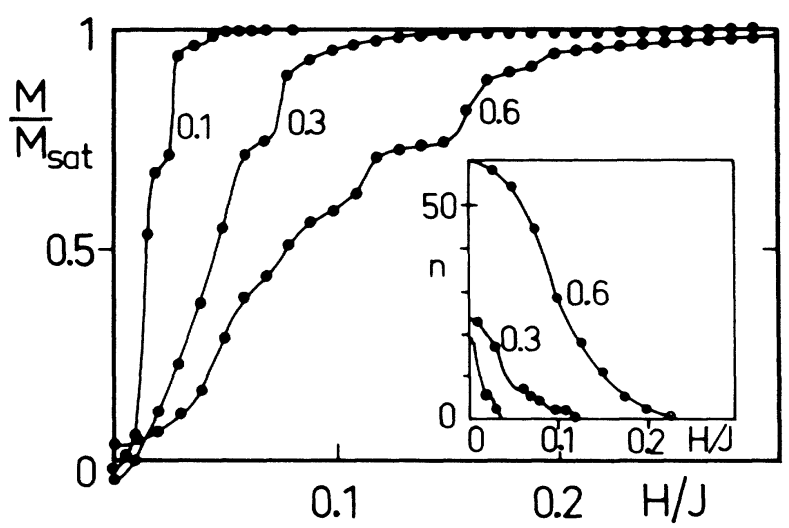

FIG. 5. First magnetization curves for three values of $D / J$ : $0.1,0.3$, and 0.6. Inset: Number of vortices $n$ vs the applied field $H / J$ for the same values of $D / J$. 
curve. This first type of magnetization process is illustrated in Fig. 6 where a detail of the spin map has been magnified for two consecutive values of the field: $D / J=0.1$ and $h=0.025$ and 0.026 . Between these two stages of the magnetization process, a vortex-antivortex pair annihilated and another shrunk. In other respects, the number of vortices of each sign is plotted in the inset of Fig. 5 versus the applied field. As expected, the fieldinduced decrease of the number of vortices is strongly correlated to the magnetization increase. The more or less important jumps observed on the magnetization curve are a consequence of the finite size of our sample: due to the limited number of vortices in the sample, the distribution of the depinning fields is discrete instead of continuous which leads to more abrupt variations than expected in a real sample. In a macroscopic sample, this vortice-annihilating process should give rise to a specific noise, maximum when $d^{2} M / d h^{2}=0$ and vanishing at the field for which the last pair of vortices disappears. Concerning the effect of $D / J$ on the number of vortices, we have already mentioned that in zero field, a nearly linear relationship $(n \alpha D / J)$ breaks down for weak random anisotropy due to important slowing down of the dynamics when $D / J \rightarrow 0$ (Fig. 2). However, the inset of Fig. 5 shows that the application of a magnetic field of low amplitude almost does not change the number of vortices for large $D / J$ but leads to important annihilation for low
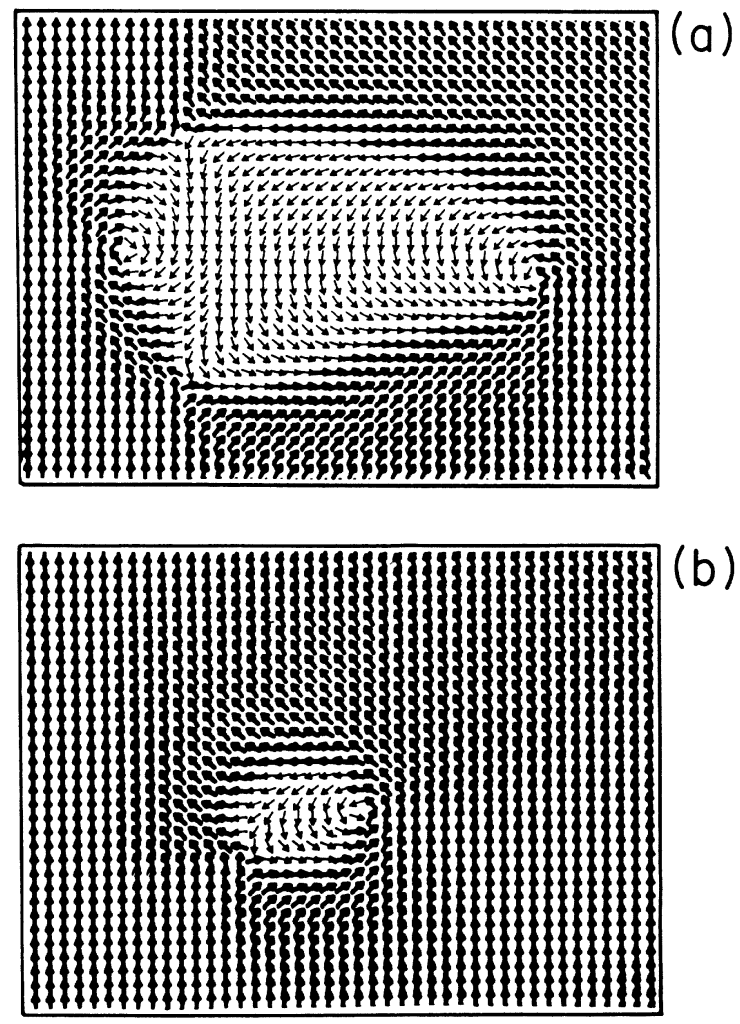

FIG. 6. Magnification of a part of the sample for $D / J=0.1$ and two consecutive values of the applied field: $H / J=0.025$ and 0.026 . Between these two stages of the magnetization process, a pair of vortices annihilated and another one shrunk.
$D / J$. This is not surprising since the pinning of vortices is due to random anisotropy. As a result, this approximately restores the linear variation $n \alpha D / J$ down to small $D / J$ values. On the other hand, the field for which the last pair of vortices disappears $\left(H_{c 1}\right)$ increases linearly with $D\left(H_{c 1} \approx 0.3 D\right)$.

(2) The second type of magnetization process of less quantitative importance than the annihilating of vortice pairs consists simply in progressive rotations of the magnetization in the intervortices area in which the magnetization was initially divided into Imry and Ma domains. These progressive rotations give a regular contribution to the magnetization curve and persist after the last vortice-pair annihilating. In the last section, we describe the hysteresis loops obtained starting from saturation.

\section{HYSTERESIS LOOPS}

The hysteresis loops obtained for three values of $D / J$ $(0.1,0.3,0.6)$ are shown in Fig. 7. Their main characteristics are a large remanent magnetization decreasing with $D / J$ but still important for $D / J=0.6$, a rapid variation of the magnetization around the coercitive field the value of which increases with $D / J$, then a kind of first saturation up to a characteristic field $H_{c 2}$ for which an abrupt jump occurs towards the true negative saturation. We note that $H_{c 2}$ is much less dependent on $D$ than the field $H_{c 1}$ characterizing the annihilating of the last pair of vortices $\left(H_{c 1} \approx 0.3 D\right)$. In particular, for weak anisotropy $(D / J<0.2), H_{c 2}$ becomes nearly independent of $D$ $\left(H_{c 2} \approx 0.28 \mathrm{~J}\right)$. We shall come back to that point later. We now go into more detail in the description of the magnetization-reversal process.

When the applied field is progressively decreased down to zero, we observe a large remanent magnetization $M_{R}$, especially for low values of $D / J$. However, the whole decreasing-field procedure is characterized by an important magnetic aftereffect. In particular, in zero field, a much longer computer run would lead to a state of lower net magnetization. Moreover, the rate of the relaxation is an increasing function of $D / J$ which means that for weak random anisotropy, the same slowing down of the dynamics occurs, as already discussed about the annihilating of vortices when starting from a completely disor-

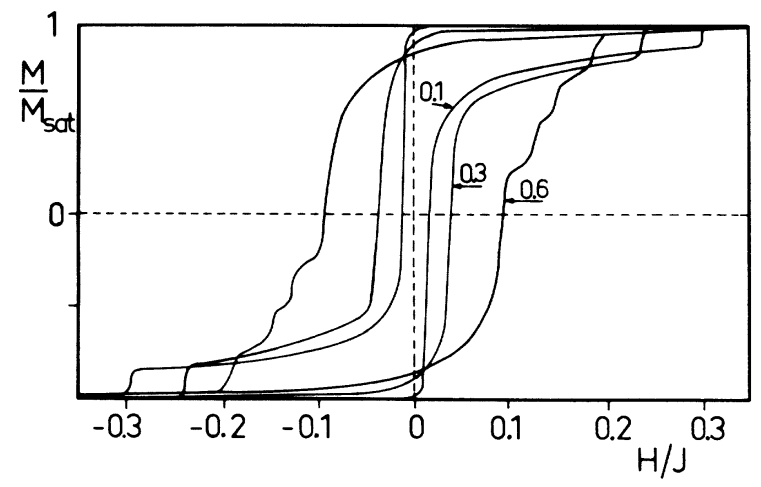

FIG. 7. Hysteresis loops obtained for three values of $D / J$ : $0.1,0.3$, and 0.6 . 
dered state. This aftereffect may be disturbing for a quantitative treatment of the values obtained for the remanent magnetization but it is not a real problem in the present qualitative discussion. Moreover, such an aftereffect is familiar in real ferromagnetic samples as soon as some pinning occurs, despite that the time scales of real experiments are several orders of magnitude longer than in computer simulations. In other respects, the rate of the relaxation is slower with two component spins (2D $X Y$ model) than with three component spins (2D Heisenberg model). The lack of the third spin component prevents rotation perpendicular to the plane of the sample. This reduces the number of energy valleys in the phase space, that the system can follow in order to lower its energy. As a result, for a same $D / J$ ratio, the remanent magnetization is found larger in the $X Y$ model than in the Heisenberg model. The spin configurations obtained here in zero field look like that of a ferromagnet with wandering axes. ${ }^{14-16}$ All the spins point in the same hemicircle defined by the direction of the saturation field. No vortices are observed in these phases.

Now if a negative field is applied, progressive rotations of blocks of moments towards the field direction occur (nucleation of domains in the opposite hemicircle, similar to the $3 \mathrm{D}$ nucleation already described ${ }^{21}$ ). Clearly enough, noncorrelated spinblocks can turn clockwise or anticlockwise depending on the local resultant anisotropy field. Between such blocks, which rotate in opposite direction, domains exist in which the spins remain in the initial direction. Figure 8 represents three successive stages of the magnetization reversal for $D / J=0.1$. They correspond to three increasing values of the negative field. However, we mention that we had observed the same kind of magnetization reversal process by applying a constant negative field of the order of the coercitive field and following the magnetization behavior versus time. In Fig. 8(a), for $h=-0.01$, nucleation of domains turning clockwise or anticlockwise can clearly be seen. Then, when the field is increased [Fig. 8(b)] (or when the field is kept to the same value but by waiting a longer time) the domains which have begun to turn, go on turning because the torque exerted by the applied field increases and becomes maximum when the magnetization of these domains passes perpendicularly to the field. On the contrary, in the area surrounded by blocks rotating in the opposite direction, where the magnetization was blocked in the initial direction, it still remains blocked because the torque exerted by the field is nearly null and the surrounding blocks tend to drive the magnetization of these areas in opposite directions. The resulting effect in these areas is a pinning of the magnetization in the initial direction of saturation. In the following of the magnetization reversal, these domains of magnetization opposite to the field play a very peculiar rule. At the early stage of their formation, it can be seen in Fig. 8(a) that some of them are isolated but others form a kind of percolating domain which extends from one side of the sample to the other with more or less numerous ramifications. The isolated domains have an elongated form and look like finite strings. They are limited at both ends by a vortexantivortex pair. Similarly, each ramification of the per-

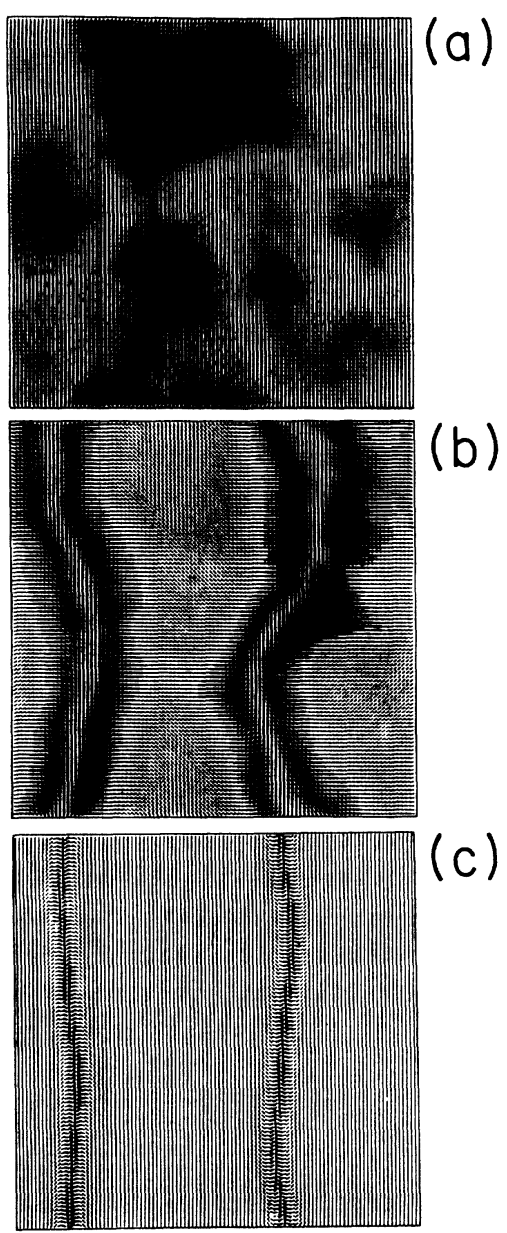

FIG. 8. Three stages of the magnetization reversal for $D / J=0.1 . \quad$ (a) $H / J=-0.01 . \quad$ (b) $H / J=-0.15$. (c) $H / J=-0.23$.

colating domain ends with a vortex of one or the other sign. Let us mention that such finite strings also exist in the first magnetization process between pairs of vortices of opposite charges. Now, when the negative field is increased, these finite strings and ramifications quickly disappear by the same process of vortices depinning, then propagation as previously discussed about first magnetization curves. As a result, when the field increases, only the percolating path of the infinite domain subsists without ramification. This percolating path constitutes the core of an infinite linear defect that we call an infinite string. When the applied field increases, this linear domain of magnetization opposite to the field shrinks by interface propagation [Fig. 8(c)]. As a result, the width of the string decreases down to a few interatomic distances for $H / J=-0.20$. These infinite strings are, in fact, static solitons. The profile of the magnetization when one crosses the string from one side to the other can be well described for weak random anisotropy, and in the continuous medium approximation by the static sineGordon equation $J\left(d^{2} \theta / d x^{2}\right)=H \sin \theta$, for which the solution is a soliton of equation:

$$
\tan \theta / 4=-\exp (-|x| / e) \text { with } e=a(J / H)^{1 / 2}
$$


(the origin of the $x$ axis is taken in the center of the defect). Thus, the width of the defect varies as $(H)^{-1 / 2}$ which is in agreement with our simulations. The string also possesses an energy per unit length

$$
E=-J \int(\nabla \theta)^{2} d x
$$

which is proportional to $(J H)^{1 / 2}$. This infinite string can be viewed as the association of two 2D Néel walls which tend to propagate in opposite directions and then compress each other when the field increases. The path followed by the core of the infinite string is the result of a competition between two phenomena: as the energy of the string increases with its length $\left[(J H)^{1 / 2}\right.$ by unit length], it tends to be as straight as possible. But the effect of the random anisotropy is to pin the string in the shape it acquires in the first stage of its formation. As a result, the weaker is the random anisotropy, the more straight is the defect, as is illustrated in Fig. 9.

Thus, when the negative field increases up to $H_{c 2}$, this kind of first saturation in the hysteresis cycle (Fig. 7) is
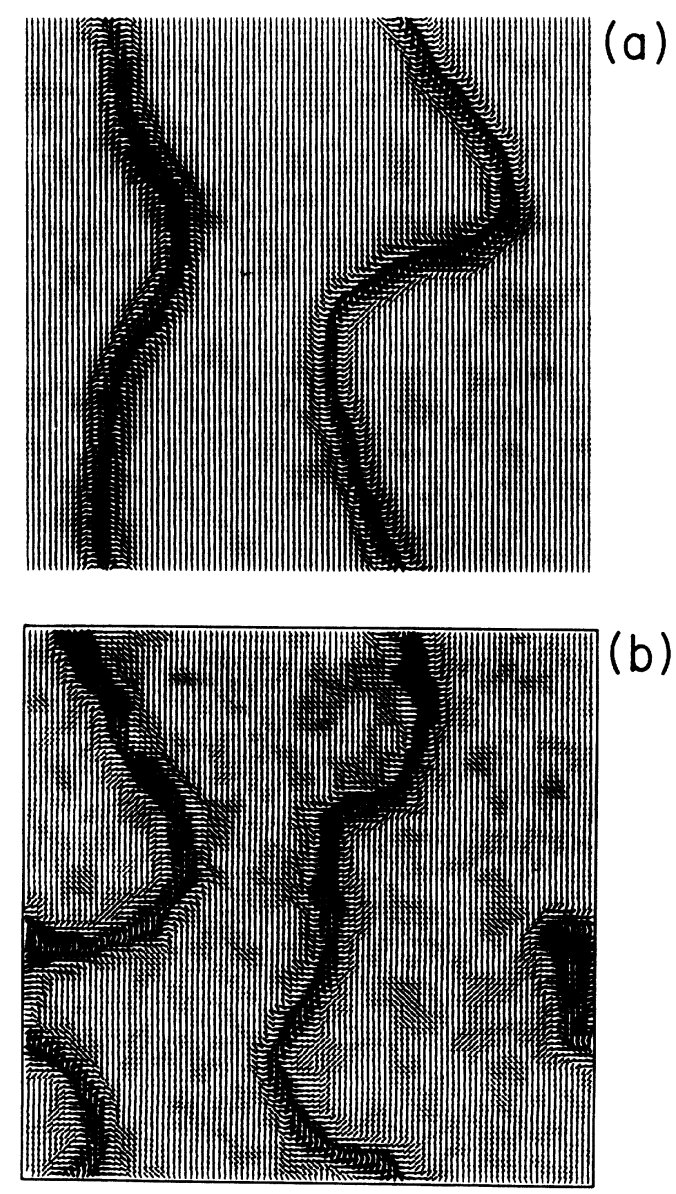

FIG. 9. Different shapes of the infinite strings for the same applied field but two different values of $D / J$ : 0.3 and 0.6 . Note that the weaker the anisotropy is, the more straight is the string. Compare also with Fig. 8(c). due to the shrinking of the string down to approximately four interatomic distances.

Then, for a critical value of the applied field $H_{c 2}$, the string abruptly collapses. In the simulation, we observe that the string begins breaking at the point where it is the most symmetrical with respect to the field with a symmetry point located on a bound between two neighboring spins. It breaks by the creation of a vortex-antivortex pair, each vortex propagating in opposite directions along the string. This collapse leads to a rather abrupt jump of magnetization in the hysteresis loop. We underline that the value of $H_{c 2}$ is independent of $D$ for weak random anisotropy. It is an intrinsic value characteristic of the field-induced collapse of the infinite string. On the contrary, the field $H_{c 1}$ which characterizes the disappearing of the last vortice pair in the first magnetization process is associated with a depinning phenomenon and thus depends on the strength of the random anisotropy $D$. This constitutes an important difference between these two characteristic fields.

Finally, we mention that such infinite linear defects can exist in the pure classical 2D $X Y$ model without any disorder. In that case, their cores are perfect straight lines at zero temperature. The cross sections of these defects are static solitons or $2 \pi$ Néel walls. In the classical $X Y$ chain, the static and dynamic properties of such topological defects have already been studied by various authors. ${ }^{20,22}$ In particular, Etrich et al. have studied the behavior of static solitons on a discrete ferromagnetic spin chain with a symmetry-breaking field. They showed that two statics in plane soliton structures exist: one with its center located on a lattice site ["central-spin" configuration (CS)] an the other with its center located in the middle between two neighboring lattice sites ["central-band" configuration (CB)]. The critical value of the applied for which the soliton breaks down is $H_{\mathrm{CS}}=0.5000 \mathrm{~J}$ for the CS soliton, $H_{\mathrm{CB}}=0.2723 \mathrm{~J}$ for the CB soliton. In our results, it can be seen in Fig. 8(c) or 9 that along an infinite string both kinds of solitons exist linked by intermediate structures. Moreover, since $H_{\mathrm{CB}}$ is lower than $H_{\mathrm{CS}}$, it is not surprising that the strings break down at a point where their cross section is a CB soliton. The critical value $H_{c 2} \sim 0.28 \mathrm{~J}( \pm 0.1)$ characterizing the breaking down of the string for vanishing random anisotropy is in good agreement with the value of $H_{\mathrm{CB}}=0.2723$ of Ref. 22. However, in the presence of random anisotropy, these defects appear naturally in a hysteresis cycle due to nucleation of domains rotating in opposite directions. Without disorder, a global rotation of the whole magnetization as a rigid block is expected when a negative field is applied on the sample so that no string should appear. However, at finite low temperature, thermal activation may nucleate some domains rotating in opposite directions as the random anisotropy does at $0 \mathrm{~K}$. Another way to obtain such strings would be to apply on the sample both a homogeneous field which maintains the whole magnetization in its direction, and a local field only on a narrow strip of the sample. The object of this local field, opposite to the homogeneous one, would be to create a local reversal of the magnetization on a width smaller than $1 \mu \mathrm{m}$. 


\section{CONCLUSION}

In this numerical study of the classical 2D $X Y$ model at zero temperature with weak random anisotropy, we focus on the magnetization behavior in the first magnetization process and hysteresis cycle. In zero field, we start from a state of zero net magnetization characterized by the presence of pinned vortices and Imry and Ma domains. Then applying an increasing magnetic field leads to a progressive depinning and annihilating of vortices, together with progressive rotations of the magnetization inside Imry and $\mathrm{Ma}$ domains towards the direction of the field. Coming from saturation and decreasing the field leads to a state of large remanent magnetization but characterized by an important aftereffect. When a negative field is applied, a new kind of linear defect (infinite strings) appears. They are a striking consequence of topological barriers in the $X Y$ model. The width of the strings decreases down to a few interatomic distances when the strength of the field increases. Then they collapse through the creation of vortex-antivortex pairs, which separate and propagate in opposite directions along the strings. This magnetization process leads to a peculiar hysteresis loop shape, narrow around the zero-magnetization axis and fairly large near saturation. To our knowledge, such linear defects have never been characterized in a real sample. However, we believe that they can be observed, for instance, in thin ferromagnetic films with strong planar anisotropy. By applying local fields (as in magnetic recording) on parallel strips of the sample, it should be possible to create parallel ferromagnetic domains of opposite magnetization separated by Néel walls. Then, by applying a magnetic field along one of the two magnetization directions, one kind of domain will shrink by propagation of the Néel walls. The linear defect discussed in this paper would correspond to the ultimate stage in this shrinking process. We believe that the dipolar effect (not taken into account in the simulation) should quantitatively change the behavior of such defects (it is well known that dipolar charges play an important role on the properties of Néel walls) but should not drastically affect the qualitative discussion of this paper.

\section{ACKNOWLEDGMENTS}

We wish to gratefully acknowledge Eugène Chudnovsky who was present while this work was in progress and strongly encouraged it by fruitful discussions. We also thank Rammal Rammal and Jacques Villain for their stimulating remarks.
${ }^{1}$ N. D. Mermin, Phys. Rev. 176, 250 (1968).

${ }^{2}$ N. D. Mermin and H. Wagner, Phys. Rev. Lett 17, 1133 (1966).

${ }^{3}$ X. Hohenberg, Phys. Rev. 158, 383 (1967)

${ }^{4} J$. M. Kosterlitz and D. J. Thouless, J. Phys. C 6, 1181 (1973); J. M. Kosterlitz, ibid. 7, 1046 (1974); J. M. Kosterlitz and D. J. Thouless, in Progress in Low Temperature Physics, edited by D. F. Brewer (North-Holland, Amsterdam, 1978), Vol. VII B, p. 373.

${ }^{5}$ For a review, see K. J. Strandburg, Rev. Mod. Phys. 60, 161 (1988).

${ }^{6}$ R. H. Swendsen, Phys. Rev. Lett. 49, 1302 (1982).

${ }^{7}$ D. H. Lee and G. Grinstein, Phys. Rev. Lett. 55, 541 (1985).

${ }^{8}$ M. Rubinstein, B. Shraimen, and D. R. Nelson, Phys. Rev. B 27, 1800 (1982).

${ }^{9}$ A. Houghton, R. D. Kenway, and S. C. Ying, Phys. Rev. B 22, 298 (1981).

${ }^{10}$ J. L. Lardy and S. Ostlund, Phys. Rev. B 25, 6899 (1982).

${ }^{11}$ Y. Y. Goldschmidt and B. Schaub, Nucl. Phys. B 251, 77 (1985).
${ }^{12}$ Y. Imry and S. Ma, Phys. Rev. Lett. 35, 1399 (1975).

${ }^{13}$ R. A. Pelcovits, E. Pytte, and J. Rudnick, Phys. Rev. Lett. 40, 476 (1978).

${ }^{14}$ E. M. Chudnovsky, J. Magn. Magn. Mater. 40, 21 (1983).

${ }^{15}$ E. M. Chudnovsky, J. Appl. Phys. 64, 5770 (1988).

${ }^{16}$ E. M. Chudnovsky, W. M. Saslow, and R. A. Serota, Phys. Rev. B 33, 1 (1986); 33, 251 (1986).

${ }^{17}$ J. Tobochnik and G. V. Chester, Phys. Rev. B 20, 3761 (1979).

${ }^{18}$ J. J. Rhyne, IEEE Trans. Magn. MAG-21, 1990 (1985).

${ }^{19}$ B. Barbara, B. Dieny, A. Liénard, J. P. Rebouillat, B. Boucher, and J. Schweizer, Solid State Commun. 55, 5 (1985); 55, 463 (1985).

${ }^{20}$ For a review, see R. W. Gerling, Comput. Phys. Rep. (Netherlands) 7, 73 (1988).

${ }^{21}$ B. Dieny, B. Barbara, G. Fillion, M. Maeder, and B. Michelutti, J. Phys. 48, 1030 (1987).

${ }^{22}$ C. Etrich, H. J. Mikeska, E. Magyari, H. Thomas, and R. Weber, Z. Phys. B 62, 97 (1985). 


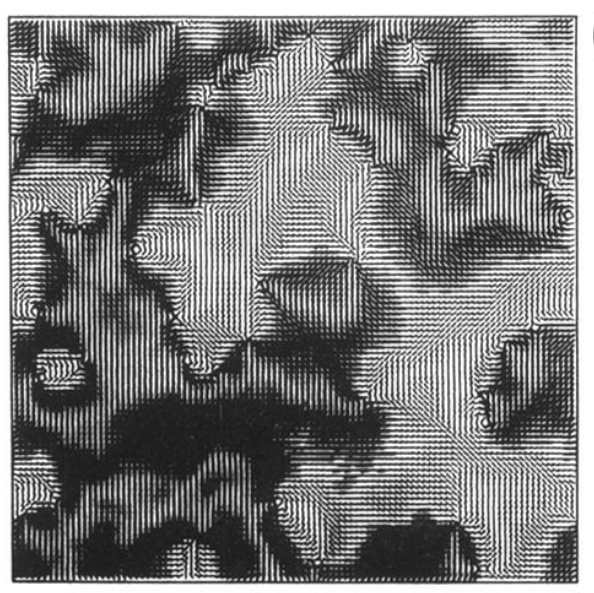

(a)

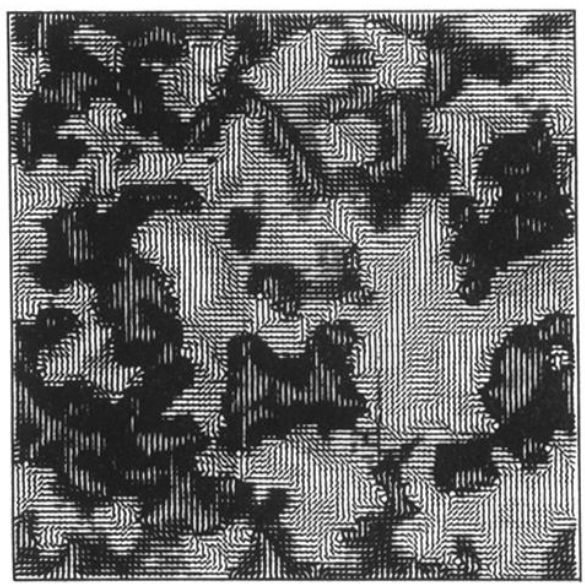

(b)

FIG. 1. Two spin configurations obtained for the same set of random-anisotropy axes but two different values of the random-anisotropy-to-exchange ratio $D / J$. (a) $D / J=0.1$. (b) $D / J=0.6$. The different thicknesses of the arrows simply indicate the spins pointing in the upper (respectively lower) hemicircle. 

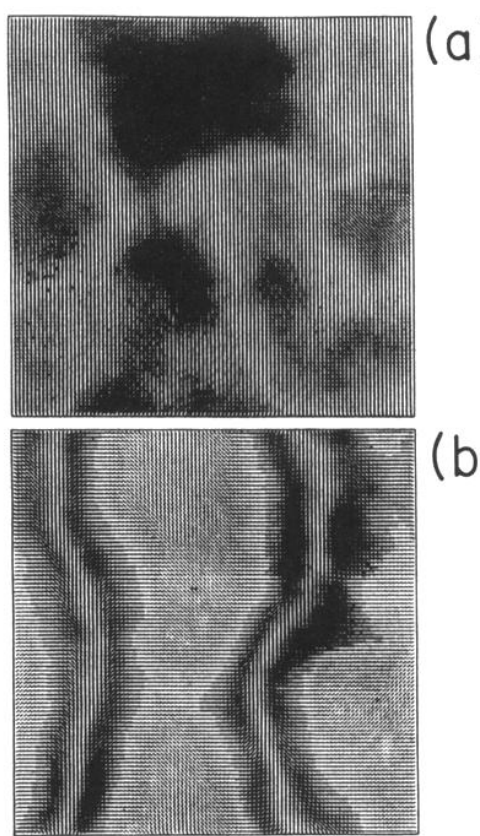

(b)

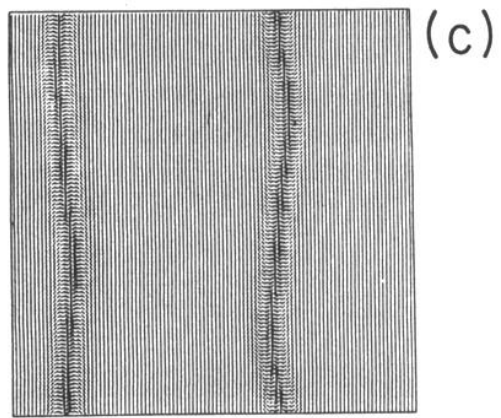

FIG. 8. Three stages of the magnetization reversal for $D / J=0.1$. (a) $H / J=-0.01 . \quad$ (b) $H / J=-0.15$. (c) $H / J=-0.23$. 


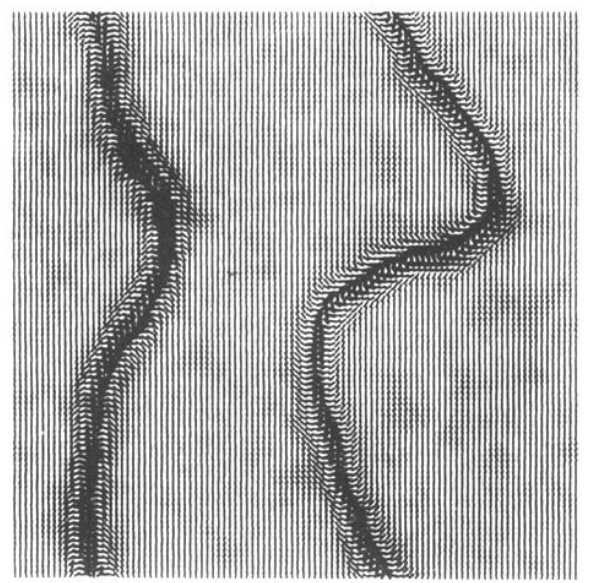

(a)

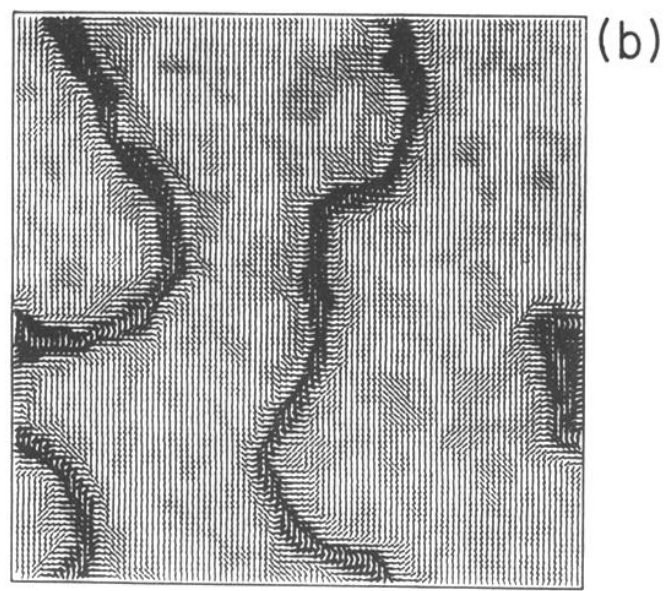

FIG. 9. Different shapes of the infinite strings for the same applied field but two different values of $D / J: 0.3$ and 0.6. Note that the weaker the anisotropy is, the more straight is the string. Compare also with Fig. 8(c). 